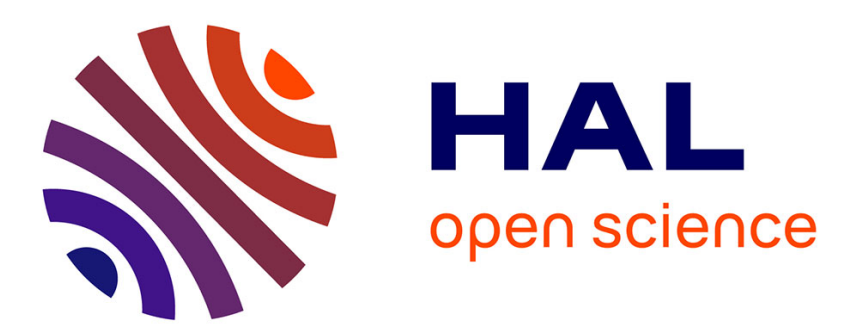

\title{
Anharmonicity and Effect of the Nanostructuring on the Lattice Dynamics of CrSi2
}

\author{
Adrien Moll, Romain Viennois, Martin Boehm, Michael Marek Koza, Yvan \\ Sidis, Jérôme Rouquette, Suzon Laborde, Jérôme Debray, Bertrand Menaert, \\ John-Paul Castellan, et al.
}

\section{To cite this version:}

Adrien Moll, Romain Viennois, Martin Boehm, Michael Marek Koza, Yvan Sidis, et al.. Anharmonicity and Effect of the Nanostructuring on the Lattice Dynamics of CrSi2. Journal of Physical Chemistry C, 2021, 125 (27), pp.14786-14796. 10.1021/acs.jpcc.1c02738 . hal-03366420

\section{HAL Id: hal-03366420 https://hal.science/hal-03366420}

Submitted on 7 Oct 2021

HAL is a multi-disciplinary open access archive for the deposit and dissemination of scientific research documents, whether they are published or not. The documents may come from teaching and research institutions in France or abroad, or from public or private research centers.
L'archive ouverte pluridisciplinaire HAL, est destinée au dépôt et à la diffusion de documents scientifiques de niveau recherche, publiés ou non, émanant des établissements d'enseignement et de recherche français ou étrangers, des laboratoires publics ou privés. 


\title{
Anharmonicity and Effect of the Nanostructuring on the Lattice Dynamics of $\mathrm{CrSi} 2$
}

\author{
A. Moll ${ }^{1}$, R. Viennois ${ }^{1}$, M. Boehm ${ }^{2}$, M. Koza ${ }^{2}$, Y. Sidis $^{3}$, J. Rouquette ${ }^{1}$, S. Laborde ${ }^{1}$, J. Debray ${ }^{5}$, B. \\ Menaert $^{5}$, J.-P. Castellan ${ }^{3}$, C. Candolfi ${ }^{4}$, B. Lenoir ${ }^{4}$, P. Hermet ${ }^{1}$, M. Beaudhuin ${ }^{1}$
}

\author{
${ }^{1}$ ICGM, Univ. Montpellier, CNRS, ENSCM, Montpellier, France \\ 2 Institut Laue-Langevin, 71 avenue des Martyrs, CS 20156, 38042 Grenoble Cedex 9, France \\ ${ }^{3}$ Université Paris-Saclay, CNRS, CEA, Laboratoire Léon Brillouin, 91191, Gif-sur-Yvette, France \\ ${ }^{4}$ Institut Jean Lamour, Université de Lorraine, CNRS, Nancy, France \\ ${ }^{5}$ Univ. Grenoble Alpes, CNRS, Grenoble INP, Institut Néel, Grenoble, France
}

\begin{abstract}
Chromium disilicide $\mathrm{CrSi}_{2}$ is an interesting compound for themoelectric applications. In order to decrease its lattice thermal conductivity that mainly limits its performance, two main routes have been investigated thus far, either increasing the unit cell disorder or creating multiple interfaces through nanostructuring. Here, we explore the effect of the latter route by investigating in detail the effect of the grain size reduction and residual microstrains on the lattice dynamics and lattice thermal conductivity. The phonon dispersion curves were measured on single-crystalline $\mathrm{CrSi}_{2}$ using inelastic neutron scattering, while the generalized vibrational density of states (GVDOS) was determined on bulk and nanostructured $\mathrm{CrSi}_{2}$. All experimental results are

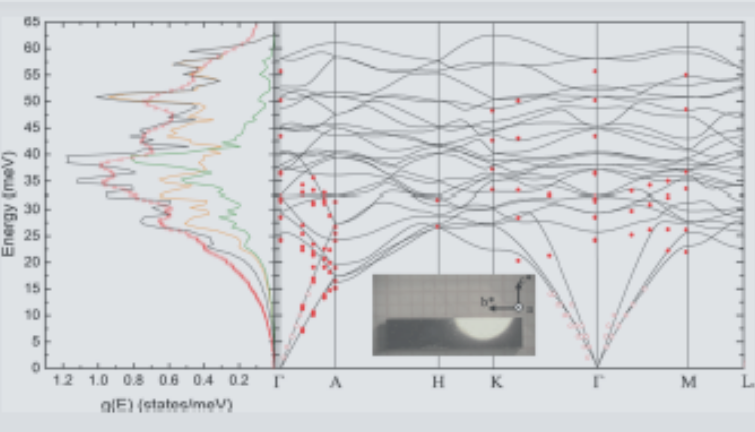
consistent with our density functional theory calculations. The results show that the optical phonons contribute from 50 to $70 \%$ of the lattice thermal conductivity. The temperature variations in the GVDOS of $\mathrm{CrSi}_{2}$ follow a quasi-harmonic behavior, which explains its rather large lattice thermal conductivity measured on the single-crystalline specimen. In addition, the GVDOS of nanocrystalline $\mathrm{CrSi}_{2}$ evidences a spectral weight transfer at low energy, which is related to a decrease in both the Debye temperature and the sound velocities that may be ascribed to an increase in both the interface density and intemal strain. These observations explain the strong decrease in the lattice thermal conductivity observed in our prior study on densified nanostructured $\mathrm{CrSi}_{2}$ pellets.
\end{abstract}

\section{INTRODUCTION}

Over the last few decades, seeking for clean and sustainable energy resources has been of growing interest due to economic and environmental concerns. Among them, thermoelectricity has been investigated with the discovery of several new promising materials. ${ }^{1}$ The performances of thermoelectric materials are determined through the dimensionless figure of ment $Z T=\frac{a^{2} \sigma T}{\lambda_{\text {let }}}$ where $\alpha$ is the thermopower $(\mathrm{V} / \mathrm{K}), \sigma$ is the electrical conductivity $(\mathrm{S} / \mathrm{m}), T$ is the absolute temperature $(\mathrm{K})$, and $\lambda_{\mathrm{mt}}=\lambda_{\mathrm{e}}+\lambda_{1}$ is the total thermal conductivity $(\mathrm{W} / \mathrm{m}$ $\mathrm{K})$, with $\lambda_{\mathrm{e}}$ and $\lambda_{1}$ being the electronic and lattice contributions, respectively. The best state-of-the-art thermoelectric materials such as $\mathrm{Bi}_{2} \mathrm{Te}_{3}, \mathrm{PbTe}_{\mathrm{e}}$, and $\mathrm{Si}_{1-x} \mathrm{Ge}_{x}{ }^{2}$ exhibit peak $Z T$ values of about $0.8-1.0$ in the room-temperature, mid-temperature, and high-temperature range, respectively. However, many elements of these materials are either expensive (Ge), scarce in the earth crust (Te), or even toxic $(\mathrm{Te} ; \mathrm{Pb})$, which requires the investigation and design of new thermoelectric materials. To overcome this issue, both $n$ - and $p$-type silicides have stirred up ever-growing interest. ${ }^{3}$ Among the silicide alloys studied thus far, several promising materials have been discovered, such as $n$-type $\mathrm{Mg}_{2} \mathrm{Si}_{1-x} \mathrm{Sn}_{x}$ (ZT up to
1.3), n-type $\mathrm{CoSi}(Z T$ up to 0.3), p-type higher manganese silicides ( $Z T$ up to 0.7$), n$ - and $p$ - type $\mathrm{FeSi}_{2}$-based alloys (ZT about $0.2-0.4$ ), and $p$-type $\mathrm{CrSi}_{2}$-based alloys ( $Z T$ up to $0.25)^{3}$ The alloys exhibit power factors $\alpha^{2} \sigma$ that rival with those obtained in $\mathrm{PbTe}$-based alloys. However, their thermal conductivity remains about one order of magnitude larger than that of $\mathrm{PbTe}$ (about $10-15 \mathrm{~W} / \mathrm{m} \mathrm{K}$ at room temperature), which strongly limits their thermoelectric performance. Thus, $\mathrm{CrSi}_{2}$-based alloys ( $\mathrm{P}_{2} 22$ space group) may become promising candidates for thermoelectric applications if their lattice thermal conductivity could be significantly lowered by alloying, nanostructuring, or a combination of both..$^{4-8}$ In this regard, we recently investigated the effect of nanostructuring on the lattice thermal conductivity of $\mathrm{CrSi}_{2}$ by mechanical ball milling and demonstrated that decreasing the grain size to 45 $\mathrm{nm}$ reduces $\lambda_{1}$ by a factor of $\sim 2 .^{6}$ Not only does this effect 
originate from an increase in the phonon scattering at the grain boundaries but also to an increase in the point-defect concentration that further scatters the propagating acoustic phonons. A better understanding of the lattice dynamics is necessary to further optimize the phonon scattering processes and reduce the thermal transport in $\mathrm{CrSi}_{2}$ to make it a competitive thermoelectric material. Using lattice-dynamics calculations of $\mathrm{CrSi}_{2}$ from the density functional theory (DFT), ${ }^{5}$ we were able to successfully assign the vibrational modes observed by Raman scattering and infrared absorption experiments. ${ }^{5,9-12}$ Our results were recently confirmed by DFT calculations of both the lattice dynamics and thermal conductivity of $\mathrm{CrSi}_{2}{ }^{13}$ However, only the optical vibrational modes at the zone center ${ }^{5,9-12}$ and the sound velocity ${ }^{14}$ have been probed so far. In this context, inelastic neutron scattering (INS) is a powerful technique as it is able to map out the phonon properties in an extended range of the energymomentum phase space. With INS on a single crystal specimen, one can map out the dispersion of the phonons, thereby setting a particular emphasis on the acoustic excitations and their possible coupling with low-energy, dispersionless optical modes. ${ }^{15-17}$ Complementary to these measurements, powder INS is the technique of choice to study the overall spectral density of phonons and is thus best suited to elaborate the impact of nanostructuring on the vibrational properties of a given material. ${ }^{18,19}$ However, combining both techniques on specific thermoelectric materials has been only scarcely carried out to date. Herein, we report for the first time the experimental phonon dispersion curves of single-crystalline $\mathrm{CrSi}_{2}$ and the generalized vibrational density of states (GVDOS) of bulk and nanocrystalline $\mathrm{CrSi}_{2}$. The INS results are supported by DFT calculations and further complemented by low-temperature measurements of the lattice thermal conductivity of single-crystalline $\mathrm{CrSi}_{2}$ perpendicular to the crystallographic $c$ axis (which corresponds to the optical axis).

\section{MATERIALS AND METHODS}

2.1. Sample Preparation. A centimeter-sized $\mathrm{CrSi}_{2}$ single crystal (named hereafter So) was elaborated using the vertical gradient freeze (VGF) method, as described in detail in our previous study, ${ }^{20}$ using a $\mathrm{CrSi}_{2}$ precursor obtained by arc melting from a stoichiometric amount of $\mathrm{Cr}$ granules (Alfa Aesar, 99.99\%) and Si lumps (Alfa Aesar, 99.9999\%) under an Ar atmosphere (Air Product, 5.2 N). The crystal was grown in a pyrolytic boron nitride crucible with a growth rate fixed to 10 $\mathrm{mm} / \mathrm{h}$ and a thermal gradient of $0.6 \mathrm{~K} / \mathrm{mm}$ under Ar.

$\mathrm{CrSi}_{2}$ powders were prepared from the $\mathrm{CrSi} i_{2}$ precursor made by arc melting as described above. Three types of samples were prepared: a micrometric powder obtained by rough crushing of the $\mathrm{CrSi}_{2}$ precursor in an agate mortar (named $\mathrm{S} 1$ ); a nanocrystalline powder ball milled during $40 \mathrm{~min}$ (S2); and a nanocrystalline powder ball milled during $240 \mathrm{~min}$ (S3).

The ball milling was performed using a Fritsch "Pulverisette 7 " planetary micromill with a silicon carbide jar $(45 \mathrm{~mL})$ and five $15 \mathrm{~mm}$ diameter balls. The powder-to-mass ratio was fixed to $1: 10$, and the speeds of the supporting disc and the grinding jar were 575 and $1150 \mathrm{rpm}$, respectively. Special attention was paid to avoid the formation of a native oxide layer at the surface of the powders. To this end, all the steps of the synthesis procedure from mortar crushing to ball milling were made in a glovebox under Ar. The powders were then sealed in silica tubes under vacuum for their transfer to the sample holders used for the INS measurements, which were sealed under argon in a glovebox. No contact of the samples with air occurred from crushing to the end of the measurement.

2.2. Microstructural Characterization. The crystal quality of sample S0 was characterized in our prior study ${ }^{20}$ by electron back scattering diffraction (EBSD) with a CamScan Crystal Probe X500FE SEM equipped with an EDX X-MaxN $20 \mathrm{~mm}^{2}$ detector and HKL NordlysNano EBSD detector. The chemical composition was determined by electron probe micro analysis (EPMA) using a CAMECA $\mathrm{SX}-\mathrm{A} 00$ instrument equipped with five wavelength-dispersive $\mathrm{X}$-ray spectrometers at the Service Microsonde Sud (Montpellier, France) on 70 points with two standards ( $\mathrm{Si}$ on Wollas; $\mathrm{Cr}$ on $\left.\mathrm{Cr}_{2} \mathrm{O}_{3}\right)$. Half of the crystal was cut into a bar-shaped sample $\left(2 \times 2 \times 12 \mathrm{~mm}^{3}\right)$ oriented along the $b^{*}$ axis (perpendicular to the $c$ axis of the crystal structure) for thermal conductivity measurements between 5 and $300 \mathrm{~K}$. The crystal was not large enough to characterize the thermal conductivity along the $c$ axis.

A single-crystalline specimen of So $(\sim 0.092 \mathrm{~mm} \times 0.165$ $\mathrm{mm} \times 0.165 \mathrm{~mm}$ ) was also used for X-ray diffraction (XRD) analysis performed using a Bruker D8 Venture diffractometer at $300 \mathrm{~K}$ (Mo source: $\mathrm{K}_{a 1}=0.7093 \AA, \mathrm{K}_{a 2}=0.7136 \AA$ ). The total exposure time was $2.06 \mathrm{~h}$. The frames were integrated with the Bruker SAINT software package using a narrow-frame algorithm. ${ }^{21}$ The integration of the data using a hexagonal unit cell yielded a total of 7271 reflections to a maximum $\theta$ angle of $53.54^{\circ}$ ( $0.44 \AA$ resolution) of which 433 were independent (average redundancy 16.792, completeness $=95.1 \%, R_{\text {int }}=$ $5.89 \%$, and $\left.R_{\mathrm{sig}}=3.33 \%\right)$ and $399(92.15 \%)$ were greater than $2 \sigma\left(\mathrm{F}^{2}\right)$. The final unit cell parameters $a=4.4289$ (2) $\AA, c=$ $6.3663(3) \AA$, and Volume $=108.146(11) \AA^{3}$ were inferred from the refinement of the XYZ-centroids of 2991 reflections above $20 \sigma(\mathrm{I})$ with $21.54^{\circ}<2 \theta<138.3^{\circ}$. Data were corrected for absorption effects using the multiscan method (SADABS). ${ }^{22}$ The ratio of minimum to maximum apparent transmission was 0.775 .

The crystal structure was solved and refined using the Bruker SHELXTL software package, ${ }^{23}$ with $Z=3$ for the formula unit, $\mathrm{CrSi} i_{2}$. The final anisotropic full-matrix leastsquares refinement on $\mathrm{F}^{2}$ with nine variables converged at $\mathrm{R} 1=$ $4.19 \%$, for the observed data and wR2 $=10.97 \%$ for all data. The goodness-of-fit is 1.395. The largest peak and hole in the final difference electron density were $1.516 \mathrm{e}^{-} / \AA^{3}$ and -1.786 $\mathrm{e}^{-} / \AA^{3}$, respectively, with an RMS deviation of $0.483 \mathrm{e}^{-} / \AA^{3}$. From this final model, the calculated density was $4.983 \mathrm{~g} / \mathrm{cm}^{3}$ and $\mathrm{F}(000), 156 \mathrm{e}^{-}$. The atomic Wyckoff positions are $(1 / 2,1 /$ $2,1 / 6)$ for the $\mathrm{Cr}$ atoms and $(0.16559(5), 0.33118(11), 0.5)$ for the $\mathrm{Si}$ atoms. The isotropic atomic displacement parameters are $U_{\text {iso }}=0.00486(13) \AA^{2}$ for the $\mathrm{Cr}$ atoms and $U_{\text {ino }}=$ $0.00661(16) \AA^{2}$ for the $\mathrm{Si}$ atoms.

Samples S2 and S3 were observed using a JEOL 2200 highresolution transmission electron microscope (HRTEM) operating at $200 \mathrm{kV}$, equipped with a CCD camera (GATAN USC) with $4092 \times 4092$ pixels $^{2}$. The phase composition of these three samples was measured from XRD analysis using a Philips X'PERT apparatus ( $\mathrm{Cu}-\mathrm{K} \alpha$ radiation $1.5406 \AA$ ) with an accelerated detector PW3050/60 at $45 \mathrm{kV}$ and $30 \mathrm{~mA}$. The crystallite sizes and strain were determined from Rietveld refinement with the FullProf software using the microstructural analysis method. ${ }^{24}$

2.3. Inelastic Neutron Scattering. Time-of-flight INS experiments were carried out on $\mathrm{CrSi}_{2}$ powders (samples $\mathrm{S} 1$, $\mathrm{S} 2$, and S3) using the thermal and cold neutron spectrometers 

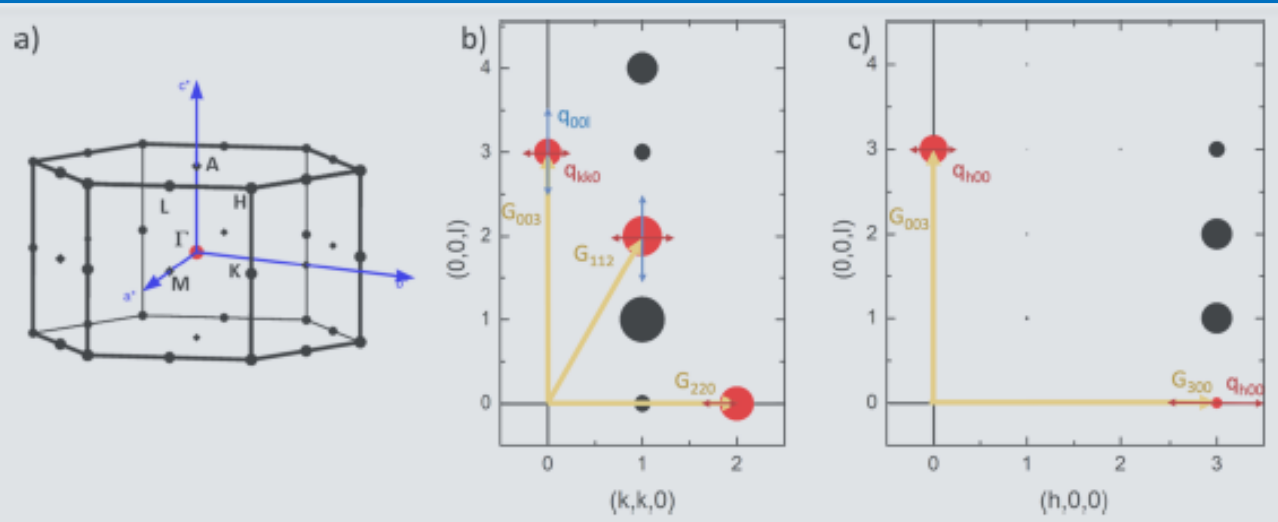

Figure 1. (a) Brillouin zone of $\mathrm{CrSi}_{2}$ and Bragg peak intensities in the scattering planes (b) $\{[110][001]\}$ and (c) $\{[100][001]\}$. The high symmetry points correspond to $\Gamma(0,0,0), \mathrm{M}(1 / 2,0,0), \mathrm{L}(1 / 2,0,1 / 2), \mathrm{A}(0,0,1 / 2), \mathrm{K}(1 / 3,1 / 3,0)$, and $\mathrm{H}(1 / 3,1 / 3,1 / 2)$. The size of the Bragg peaks in (b) and (c) is proportional to the nuclear elastic structure factor. The scanning direction along the Bragg Peaks (330), (112), (220), and (003) is indicated with the reciprocal lattice vector $\vec{G}$ and the reduced wave vector $\vec{q}$ such as the wave vector $\vec{Q}=\vec{G}+\vec{q}$.

IN4 and IN5, respectively, of the European neutron source Institut Laue-Langevin in Grenoble, France. On the IN4 spectrometer, an incident neutron wavelength of $1.1 \AA$ was applied covering the elastic wave vector $Q$ range of $1.5-10 \AA^{-1}$ with an energy resolution of $4.8(1) \mathrm{meV}$ at the elastic peak. On the IN5 spectrometer, a wavelength of $5 \AA$ was chosen to access an elastic $Q$ range of $0.2-2.3 \AA^{-1}$ and an elastic resolution of $0.079(2) \mathrm{meV}$. The Stokes and anti-Stokes lines were exploited for the analysis of IN4 and IN5 data, respectively.

The data were reduced using the ILL LAMP software package. ${ }^{25}$ Standard data corrections have been applied for an empty aluminum sample holder; variation in detector efficiencies and their neutron wavelength dependence and frame overlap before the signal was converted into a dynamic structure factor. The GVDOS was computed within the incoherent approximation that implies an averaging of the inelastic signal over the accessed momentum space. ${ }^{15}$ The GVDOS is normalized by 27 corresponding to the number of vibrational modes.

Triple-axis spectrometer (TAS) INS studies were further performed on single-crystalline $\mathrm{CrSi}_{2}$ (sample $\mathrm{SO}$ ) on the thermal TAS 1T at the Laboratoire Léon Brillouin in Saclay, France. The phonon dispersion was monitored along highsymmetry directions with a fixed final neutron energy of 14.7 $\mathrm{meV}\left(k_{f}=2.662 \AA^{-1}\right)$. A pyrolytic-graphite filter was inserted in the scattered neutron beam to remove higher-order contaminations.

The Brillowin zone of $\mathrm{CrSi}_{2}$ is shown in Figure 1a. Two different orientations of the crystal were exploited. The sample was aligned with the scattering plane formed by the directions: (i) $[110]$ and $[001]$ accessing the wave vectors $\vec{Q}=(112), \vec{Q}$ $=(220)$, and $\vec{Q}=(003)$ and (ii) $[100]$ and [001] accessing the wave vectors $\vec{Q}=(300)$ and $\vec{Q}=(003)$ as depicted in Figure $1 b, c$. The acoustic phonons have been measured near the nuclear Bragg peaks (112), (300), and (003) by choosing the appropriate wave vector $\vec{q}$. However, as their acoustic branches are strongly dispersive, Q-scans at constant energy were performed up to $15 \mathrm{meV}$. Above $15 \mathrm{meV}$, energy scans at constant $\vec{Q}$ were carried out near the Bragg peaks (112),
(300), $\vec{Q}=(003)$, and (220). The energy resolution was ranging between 1 and $2 \mathrm{meV}$ enabling one to record a large number of spectra in a short period of time. Indeed, $\mathrm{Cr}$ and $\mathrm{Si}$ have scattering cross-sections of only 3.47 and 2.173 , respectively. In order to extract properly the intrinsic position of a phonon, the recorded spectra were fitted using a Gaussian function for deconvolution of the peaks.

2.4. Transport Property Measurement. Low-temperature $(5-300 \mathrm{~K})$ electrical resistivity and thermal conductivity were simultaneously measured using the thermal transport option of the physical property measurement system (PPMS, Quantum Design) in the continuous measurement mode on the single-crystalline bar-shaped sample (S0) perpendicular to the $c$ direction (that is, along $b^{*}$ ). The contacts were realized by soldering four copper leads onto the sample using a lowmelting point braze. This approach allows a good thermal coupling between the sample and the leads.

2.5. Ab-Initio Calculations. DFT calculations were performed using a $2 \times 2 \times 2$ hexagonal supercell (72 atoms) using the Vienna ab initio Simulation Package (VASP). ${ }^{26,27}$ We used PAW pseudopotentials and the Perdew-BurkeEmzerhof (PBE) exchange-correlation functional. For all calculations, an energy cut-off of $450 \mathrm{eV}$ was applied. During the relaxation calculations, the forces were converged until the residual forces were less than $10^{-5} \mathrm{eV} / \AA$ and pressure less than $0.001 \mathrm{GPa}$. A full relaxation (lattice parameters and atomic positions) is reached when the residual forces and pressure were less than $1 \times 10^{-5} \mathrm{eV} / \AA$ and $1 \times 10^{-3} \mathrm{GPa}$, respectively. The obtained relaxed lattice parameters at the GGA level as parametrized by PBE, $a=4.4055 \AA$ and $c=6.3632 \AA$, are in very good agreement with the experimental results by less than $-0.5 \%$. We also tested two other exchange-correlation functionals, like PBEsol (GGA) ${ }^{28}$ and PW92 (LDA), ${ }^{29}$ but the agreement with the experimental structure was less satisfactory than for the PBE (GGA) with a relative error on the lattice parameters up to -1.4 and $-2 \%$, respectively.

Lattice dynamics calculations were performed using a $2 \times 2$ $\times 2$ hexagonal supercell ( 72 atoms) after converging the atomic positions with the same conditions than above. Dynamical matrices (harmonic and anharmonic) were built from the finite displacement method using a displacement of $0.03 \AA$ and $\Gamma$-centered $6 \times 6 \times 6 \mathrm{k}$-mesh. The phonon 


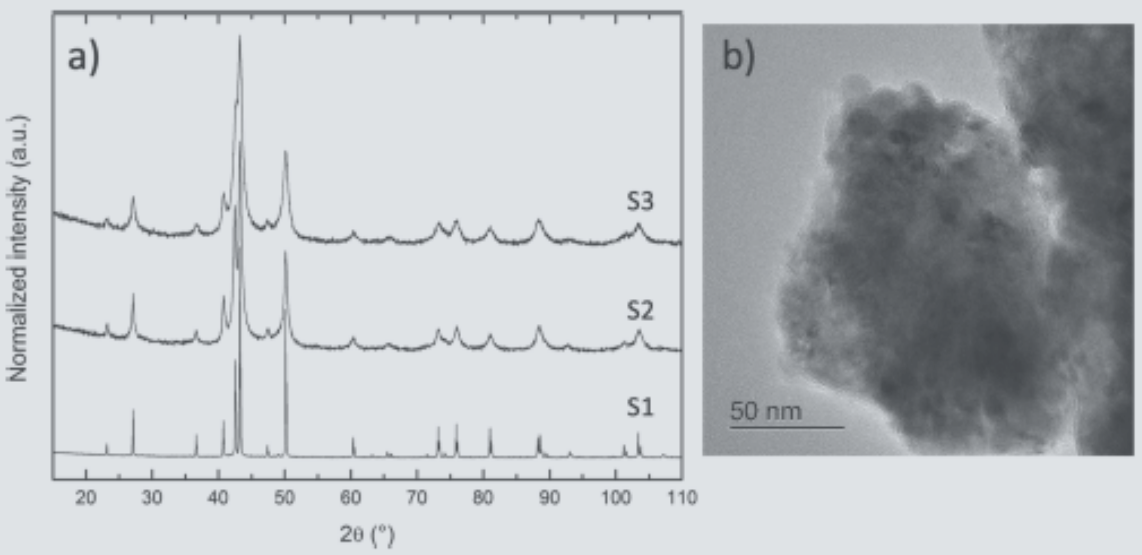

Figure 2. (a) XRD analysis of the micrometric $\mathrm{CrSi}_{2}$ powder ( $\mathrm{S} 1$ ), the nanocrystalline $\mathrm{CrSi}_{2}$ powder milled 40 min ( $\mathrm{S} 2$ ), and the nanocrystalline $\mathrm{CrSi}_{2}$ powder milled $240 \mathrm{~min}$ (S3). Each peak can be attributed to the $\mathrm{CrSi}_{2}$ phase. (b) Bright field HRTEM image of sample $\mathrm{S} 3$.

dispersion curves and phonon density of states were obtained straightforwardly by diagonalizing the dynamical matrix. For comparison with the INS data, we have calculated neutronweighted densities of states taking into account the effect of the Debye-Waller factor at different temperatures and the energymomentum phase space covered by the IN4 and IN5 spectrometers during our experiments as outlined in ref 15 . The $U_{\text {iso }}(\mathrm{T})$ values of $\mathrm{Cr}$ and $\mathrm{Si}$ were calculated from their partial densities of states derived from our DFT calculations. At $300 \mathrm{~K}$, we compute $U_{\text {iso }}=0.003705 \AA^{2}$ for the $\mathrm{Cr}$ atoms and $U_{\text {ino }}=0.00565 \AA^{2}$ for the $\mathrm{Si}$ atoms. They are slightly smaller than the experimental values, which may be expected as the static disorder present in the real compounds and monitored in experiments is not taken into account in our calculations.

From the derived second-order (harmonic) and third-order (anharmonic) constants, the lattice thermal conductivity $\lambda_{1}$ was calculated using the relaxation time approximation within the Boltzmann transport theory (see ref 30 for the details of the method). Numerically, $\lambda_{1}$ was calculated using the tetrahedron method over a $14 \times 14 \times 14$ mesh of q-points. This grid allows for reaching converged results since a $20 \times 20 \times 20 q$-mesh only leads to an increase in $\lambda_{1}$ by only $1.5 \%$ at $300 \mathrm{~K}$. In our calculations, the cartesian axis is respectively aligned along the (a, c) crystal axes, whereas the y-cartesian axis is free. We did not perform a convergence test on the size of the simulation box as a $2 \times 2 \times 2$ supercell ( 72 atoms) was the maximum size accessible by our computational resources for the calculation of $\lambda_{1}$ and this same supercell was used by Nakasawa et al. ${ }^{13}$ for similar calculations.

\section{RESULTS AND DISCUSSION}

3.1. Microstructural Characterization. As shown in our prior study, ${ }^{20}$ our large single crystal So has been characterized by EBSD, evidencing a mosaicity of $0.5^{\circ}$. Its chemical composition, determined by EPMA, is highly homogeneous with a stoichiometry determined to be $\mathrm{CrSi}_{196}$ with silicon vacancies. The refined lattice parameters $a=4.435(2) \AA$ and $c$ $=6.374(6) \AA$ are in good agreement with those determined in our previous study $(a=4.431(7) \AA$ and $c=6.365(5) \AA) .{ }^{4}$

All the peaks of the XRD patterns of the S1, S2, and S3 samples, shown in Figure $2 \mathrm{a}$, are attributed to the $\mathrm{CrSi}_{2}$ phase, evidencing their phase-pure character within the detection limits associated with this technique. The unit cell parameters of the S1 sample, inferred from Rietveld refinement, $a=4.433$ (4) $\AA$ and $c=6.366$ (4) $\AA$, are also in good agreement with our previous study. ${ }^{4}$ The micrometric $\mathrm{CrSi}_{2} \mathrm{~S} 1$ sample is well crystallized with sharp Bragg peaks, while a more pronounced broadening of the peaks is observed for the $\mathrm{CrSi} i_{2}$ precursor milled for 40 (S2) and $240 \mathrm{~min}$ (S3). The full width at half maximum (FWHM) of the peaks corresponds to crystallite sizes of 64 and $14 \mathrm{~nm}$ and microstrains of 0.75 and $0.87 \%$, respectively (see Table 1 ). ${ }^{24}$ HRTEM analysis carried out on the S3 sample is given in Figure $2 \mathrm{~b}$ and shows an average crystallite size of about $15 \mathrm{~nm}$, in agreement with the XRD observation.

Table 1. Sample List Given with their Crystallite Size and Internal Strain

$\begin{array}{ccc}\text { sample } & \text { crystallite size }(\mathrm{nm}) & \text { internal strain (\%) } \\ \text { S0 } & >1000 & \\ \text { S1 } & 64 & 0.75 \\ \text { S2 } & 14 & 0.87\end{array}$

3.2. Lattice Dynamics of Bulk $\mathrm{CrSi}_{2}$. Typical energy scans obtained with the $1 \mathrm{~T} 1$ spectrometer on the single crystal (So) are shown in Figure 3 in the $\Gamma M$ direction around $\vec{Q}=$ (300). The FWHM phonon lines are limited by the experimental resolution, and it is not possible to extract the phonon lifetimes from these energy scans. The deconvolution of this scans was made with three or four phonon lines. One notices that the intensity of the phonon lines can be close to the background noise and needs a thorough comparison with signal from different Brillouin zones and DFT calculations. This approach was used, for example, for deconvolution of the phonon line at about $33 \mathrm{meV}$ for $\Gamma=(300)$.

The experimental phonon dispersion curves and GVDOS measured by INS at $300 \mathrm{~K}$ on single-crystalline (SO) and micrometric powdered $\mathrm{CrSi}_{2}$ (S1) are shown in Figure 4. These results are compared with those obtained by DFT at 0 $\mathrm{K}$. The acoustic branches and the lowest-energy optical branches observed by INS are well reproduced by our calculations. The main structures in the experimental phonon GVDOS are consistent with the calculations. We do not observe pronounced peaks at low energies in the GVDOS, 

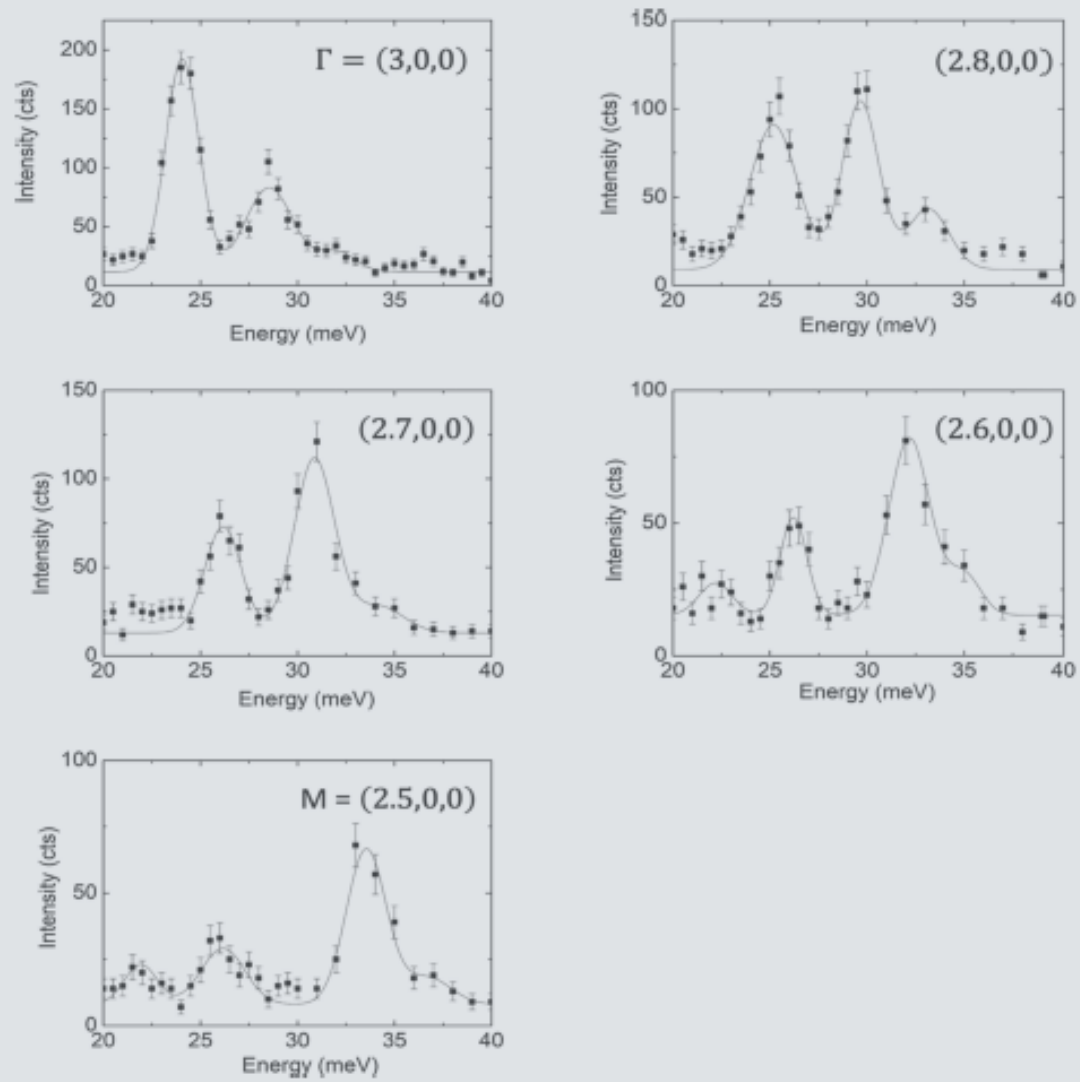

Figure 3. Enervy scan with constant $\vec{O}=(300)+\vec{a}(h 00)$ along the $\Gamma-\mathrm{M}$ direction. The solid lines are fits as explained in the Experimental Section.

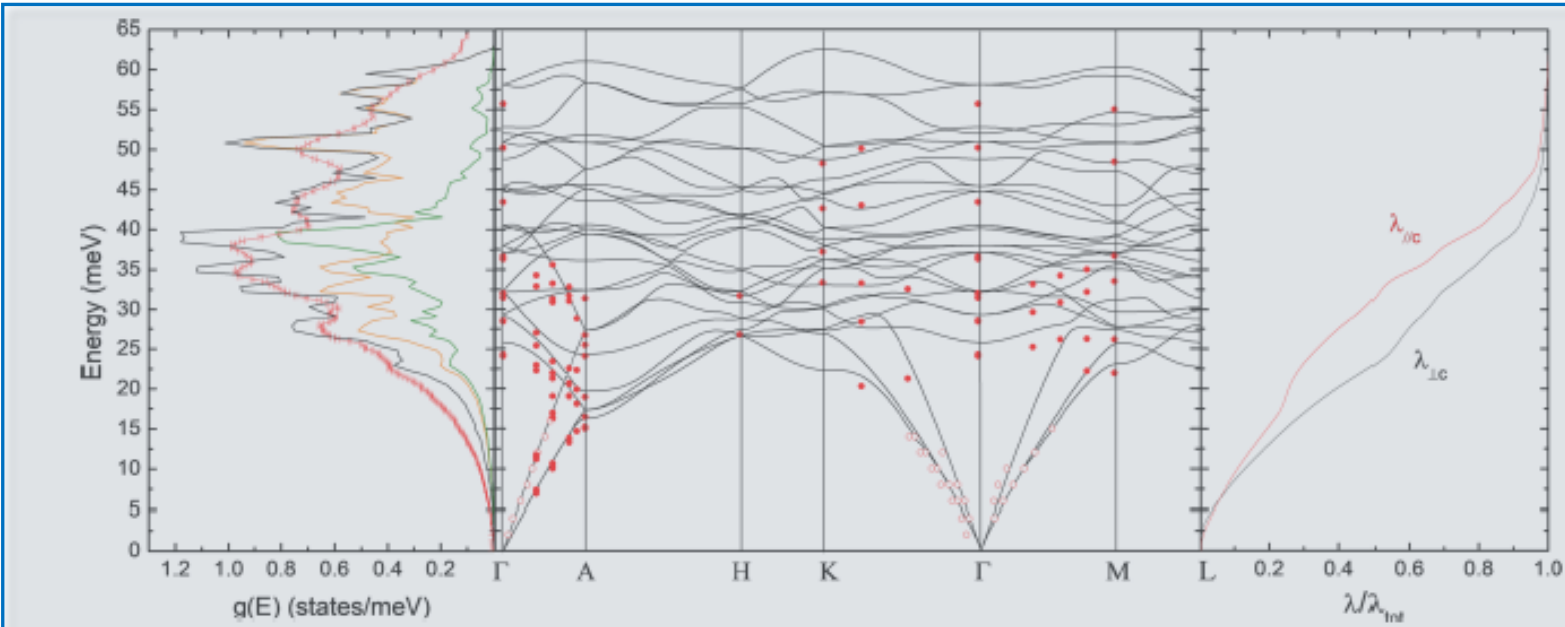

Figure 4. (left) Calculated and experimental phonon GVDOS measured on the IN5 spectrometer (black: total calculated GVDOS, orange: Si calculated contribution, green: Cr calculated contribution, and red: experimental GVDOS), (center) phonon dispersion curves determined on the 1 T1 triple-axis spectrometer at $300 \mathrm{~K}$ with hollow symbols corresponding to constant energy scans where $Q$ is scanned and filled symbols to constant $\Omega$ seane where the enerov is scanned. and (rioht) contrihution of the vihrational modes to the tatal thermal condurtivity

indicating the absence of an amorphous impurity phase as suggested in ref 31 . The optical phonons above $40 \mathrm{meV}$ are mostly due to $\mathrm{Si}-\mathrm{Si}$ bonds, while, at lower energies, the main peaks are related to $\mathrm{Cr}-\mathrm{Si}$ bonds. These experiments confirm that the lowest energy optical branches have rather large and negative dispersion along $\Gamma$-A, while they exhibit weak and positive dispersion in the $\Gamma \cdot \mathrm{K}$ and $\Gamma-\mathrm{M}$ directions. There is probably a strong interaction between the longitudinal acoustic branch and the lowest energy optical branches, especially along $\Gamma$-A where an anticrossing is observed. This means that only a 

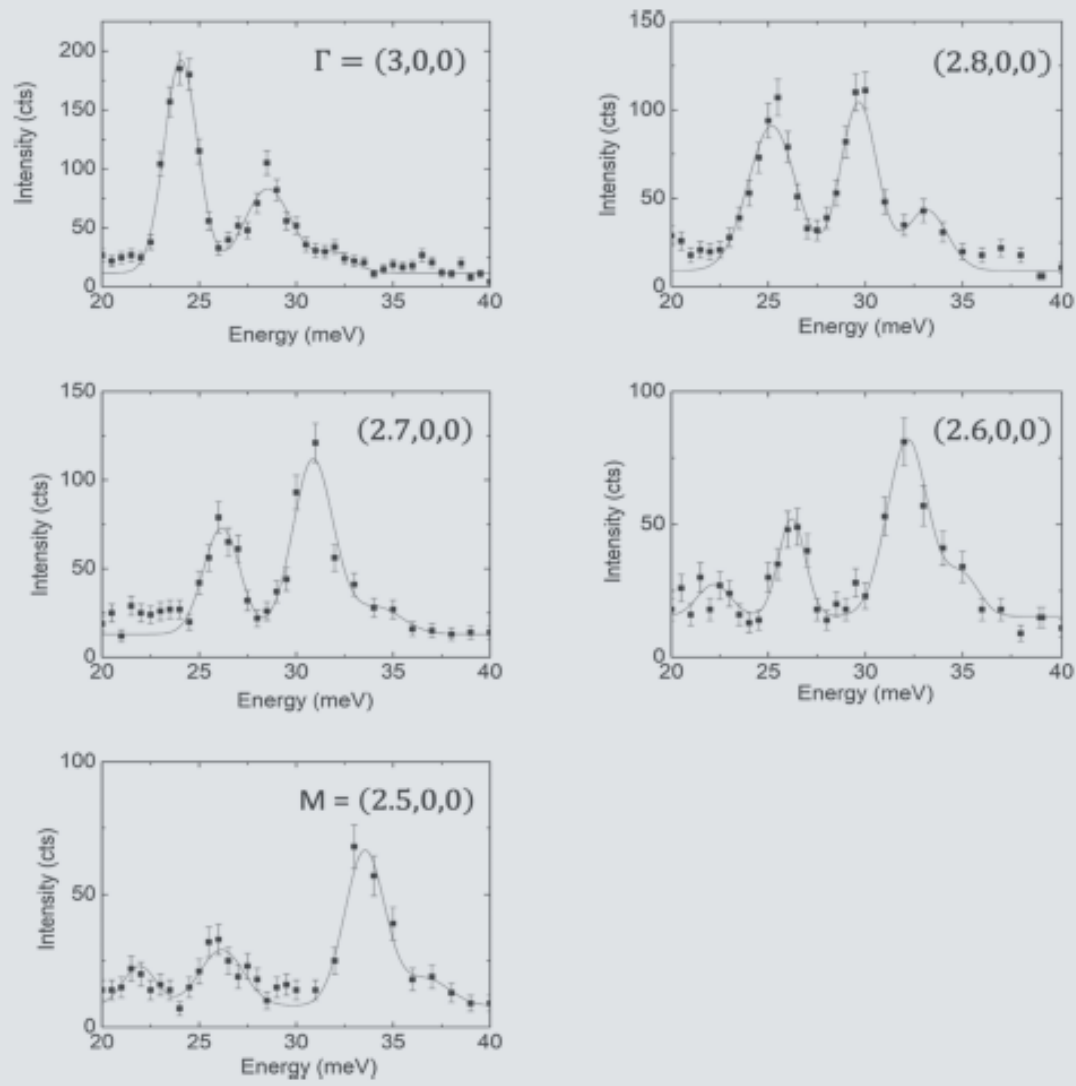

Figure 3. Energy scan with constant $\vec{Q}=(300)+\vec{q}(h 00)$ along the $\Gamma$-M direction. The solid lines are fits as explained in the Experimental Section.

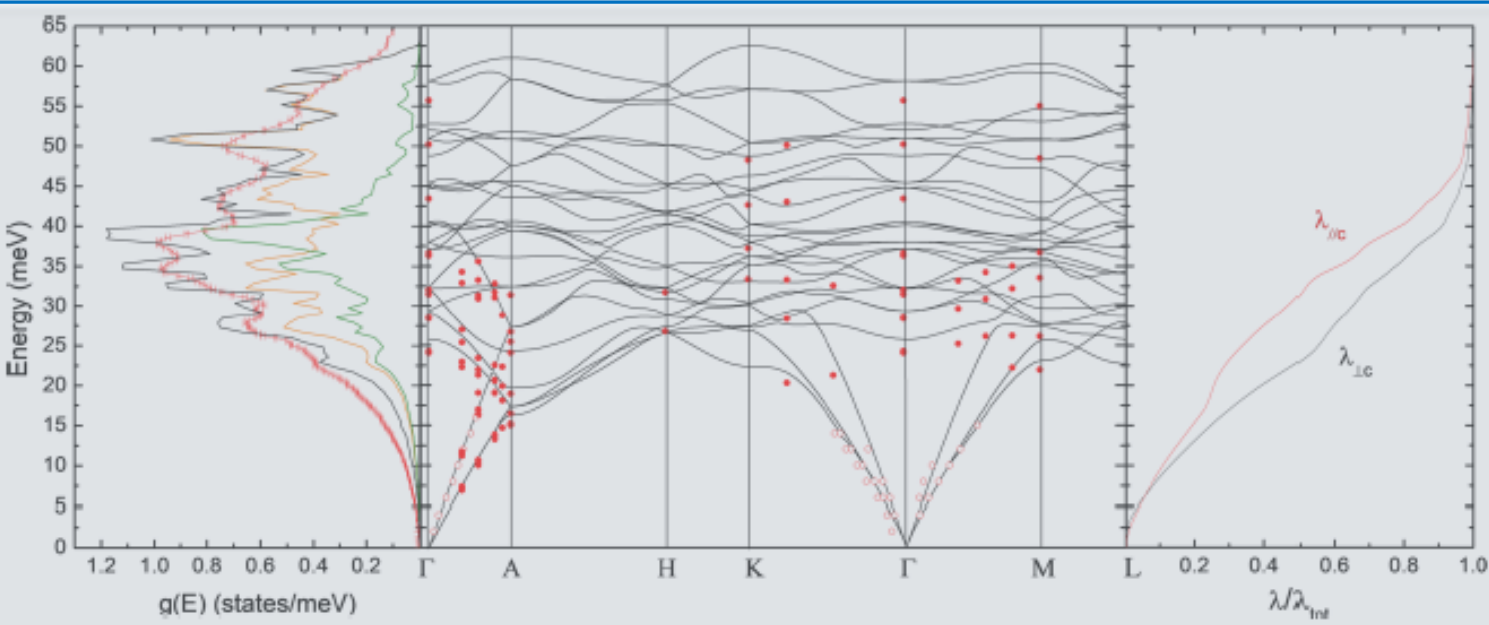

Figure 4. (left) Calculated and experimental phonon GVDOS measured on the IN5 spectrometer (black: total calculated GVDOS, orange: Si calculated contribution, green: Cr calculated contribution, and red: experimental GVDOS), (center) phonon dispersion curves determined on the 1 $\mathrm{T} 1$ triple-axis spectrometer at $300 \mathrm{~K}$ with hollow symbols corresponding to constant energy scans where $\mathrm{Q}$ is scanned and filled symbols to constant Q scans where the energy is scanned, and (right) contribution of the vibrational modes to the total thermal conductivity.

indicating the absence of an amorphous impurity phase as suggested in ref 31 . The optical phonons above $40 \mathrm{meV}$ are mostly due to $\mathrm{Si}-\mathrm{Si}$ bonds, while, at lower energies, the main peaks are related to $\mathrm{Cr}-\mathrm{Si}$ bonds. These experiments confirm that the lowest energy optical branches have rather large and negative dispersion along $\Gamma-A$, while they exhibit weak and positive dispersion in the $\Gamma-\mathrm{K}$ and $\Gamma-\mathrm{M}$ directions. There is probably a strong interaction between the longitudinal acoustic branch and the lowest energy optical branches, especially along $\Gamma$-A where an anticrossing is observed. This means that only a 

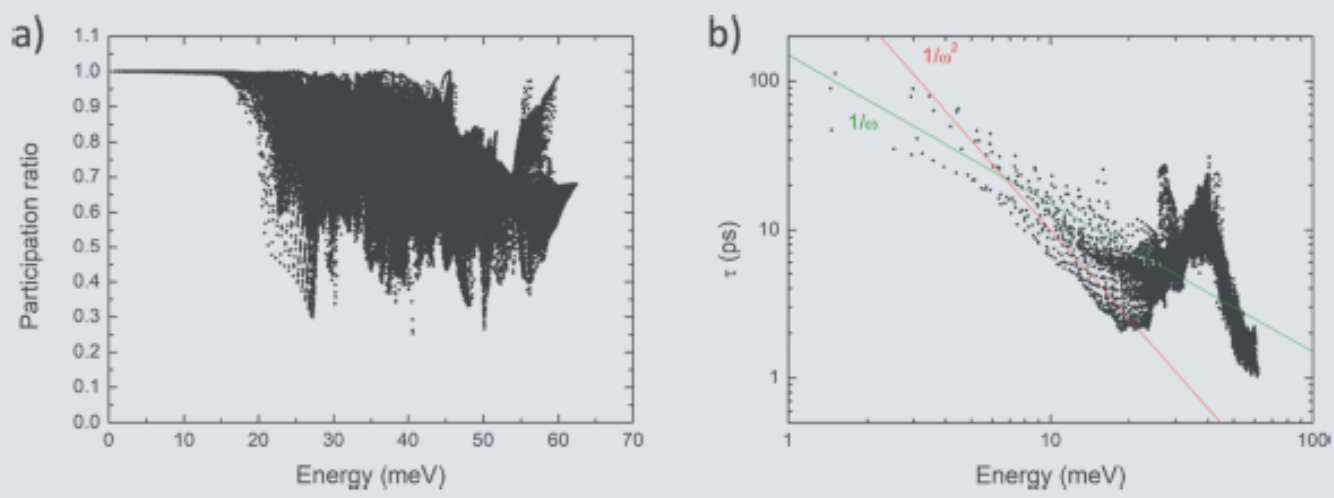

Figure 5. (a) Calculated participation ratios and (b) relaxation time of the $\mathrm{CrSi}_{2}$ phonons as a function of their energies.

limited range of the longitudinal acoustic phonons participate to the heat conduction. In contrast, the interaction of the transverse acoustic branches with the lowest energy optical branches is much weaker. The participation ratio (PR), which is reported in Figure 5a, confirms that the acoustic branches show a propagative character up to $17 \mathrm{meV}$ (i.e., with the PR close to 1). Above $17 \mathrm{meV}$, both propagative and more localized modes that have a low participation to the propagation (i.e., with a low PR) are observed. Propagative modes are also observed above $25 \mathrm{meV}$, which corresponds to optical modes with very significant dispersion.

The DFT calculation of the contributions to the total lattice thermal conductivity (Figure 4-right) shows that acoustic and optical phonons contribute nearly equally $(\sim 50 \%)$ to the total lattice thermal conductivity. The contribution of optical phonons is even larger in the $c$ direction by reaching $\sim 70 \%$ of the total lattice thermal conductivity. The optical phonons between 25 and $40 \mathrm{meV}$ contribute in that case to $\sim 35 \%$ of the total lattice thermal conductivity as there is a strong dispersion of the optical modes. Consequently, the Callaway's model, ${ }^{32}$ which is commonly used to predict the thermal conductivity considering only acoustic branches, should not be considered.

The relaxation times $\tau$ of the different phonon modes have been computed by DFT calculations and are reported as a function of the energy in Figure 5b. Below $6 \mathrm{meV}$, the relaxation time decreases as $1 / \omega$, more slowly than the expected frequency dependence $1 / \omega^{2}$ for the Umklapp scattering, ${ }^{33,34}$ which is observed between 6 and $20 \mathrm{meV}$. Below $6 \mathrm{meV}$, the relaxation time $\tau$ of the acoustic branches is much larger than that of the optical branches, suggesting a significantly stronger anharmonicity above $6 \mathrm{meV}$. However, the relaxation time for the optical branches in the $25-40 \mathrm{meV}$ range is slightly larger than the relaxation time of the acoustic branches in the $10-25 \mathrm{meV}$. This finding explains the large contribution of the optical modes to the thermal conductivity, especially in the $c$ direction where the dispersion of the optical branches is quite significant. The relaxation time of the optical modes involving motions of $\mathrm{Si}$ atoms above $50 \mathrm{meV}$ is smaller, indicative of their smaller contribution to the total thermal conductivity. The low $\tau$ of these modes is related to a broadening of the Raman FWHM compared to the FWHM of the modes at lower energies. ${ }^{5}$ As their intensity is also weak, this explains the experimental difficulties to observe the Raman-active $\mathrm{E}_{2}$ mode predicted at $448 \mathrm{~cm}^{-1}(56 \mathrm{meV}) .^{5}$
The phonon group velocity $\left(v_{g}\right)$ can be estimated as the slope of the acoustic branches near the $\Gamma$ point using the relation:

$$
v_{\mathrm{g}}=\lim _{k \rightarrow 0} \frac{1}{\hbar} \frac{d E}{d k}
$$

where $\hbar$ is the reduced Planck constant and $k$ is the wave vector. Experimental (resp. calculated) transverse (TA) and longitudinal ( $L A$ ) acoustic phonon group velocities have been obtained (see Table 2) from a fit of the experimental data

\begin{tabular}{|c|c|c|c|c|}
\hline \multirow[b]{2}{*}{ direction } & \multirow[b]{2}{*}{ acoustic branch } & \multicolumn{2}{|c|}{ experiment } & \multirow{2}{*}{$\frac{\text { DFT }}{\text { this study }}$} \\
\hline & & this study & 14 & \\
\hline \multirow[t]{2}{*}{ Г-A $(/ / c)$} & LA & $9250(280)$ & $8795(7)$ & $9120(9)$ \\
\hline & TA & $5680(220)$ & $5471(11)$ & $5980(10)$ \\
\hline \multirow[t]{2}{*}{$\Gamma-\mathrm{M}(\perp c)$} & LA & $10,270(930)$ & / & $9260(24)$ \\
\hline & TA & $6960(270)$ & / & $6560(42)$ \\
\hline
\end{tabular}

(resp. calculation) using a second-order polynomial function. Those results are in good agreement with the ultrasonic wave velocity measurements ${ }^{14}$ and our present and our previous calculations ${ }^{5}$ obtained by the density functional perturbation theory. The average sound velocity $v_{s}$ can be deduced from these data according to

$$
\frac{1}{v_{s}}=\frac{1}{3}\left[\frac{2}{v_{T A}}+\frac{1}{v_{L A}}\right]
$$

with $v_{T A}$ and $v_{L A}$ being the phonon group velocities for TA and LA branches, respectively. $v_{\mathrm{s}}$ reaches $6519 \mathrm{~ms}^{-1}$ (resp. 6755 $\mathrm{m} . \mathrm{s}^{-1}$ for PBE DFT calculation) along the $c$ axis, which is similar to that obtained by ultrasound measurements $(6260$ $\left.\mathrm{m} . \mathrm{s}^{-1}\right){ }^{14}$ Perpendicular to this axis, $v_{\mathrm{g}}$ is slightly higher and reaches $7797 \mathrm{~m} \cdot \mathrm{s}^{-1}$ (resp. $7266 \mathrm{~m} \cdot \mathrm{s}^{-1}$ for PBE DFT calculation), but no experimental data are available in the literature for this direction.

The response of phonons to temperature variations for the bulk S1 sample is presented in Figure $6 \mathrm{a}$ and Figure $6 \mathrm{~b}$. Throughout the temperature range from 1.5 to $300 \mathrm{~K}$, the overall GVDOS is not altered considerably. We observe however a moderate sharpening of distinct peaks and an 
a)

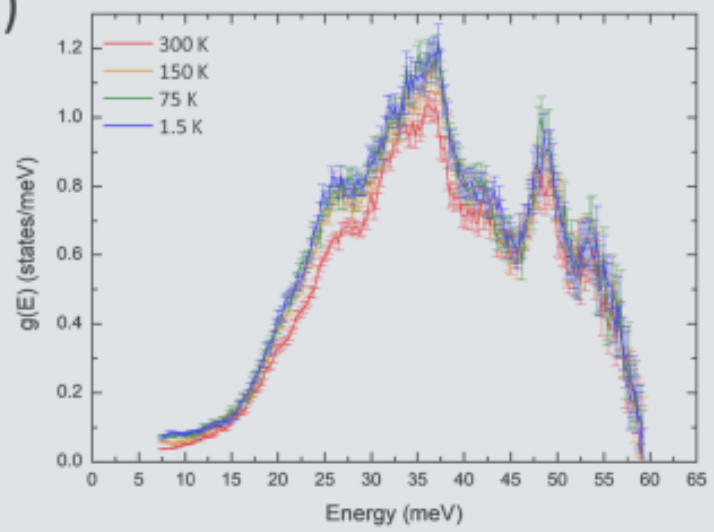

c)

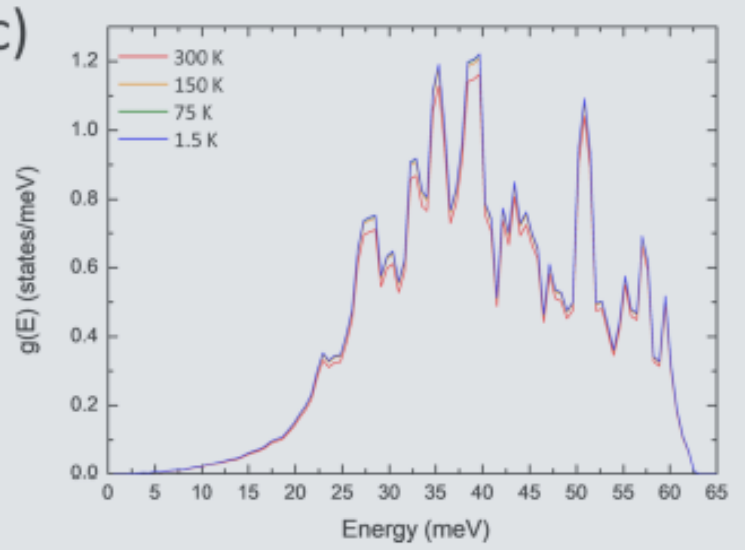

b)

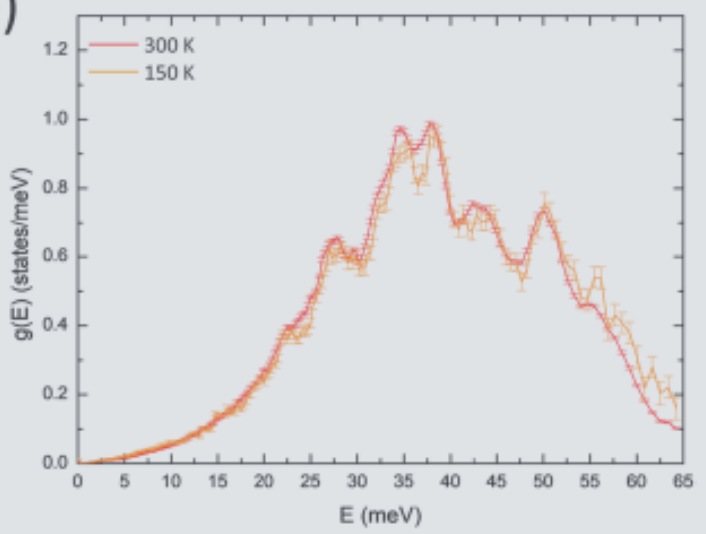

d)

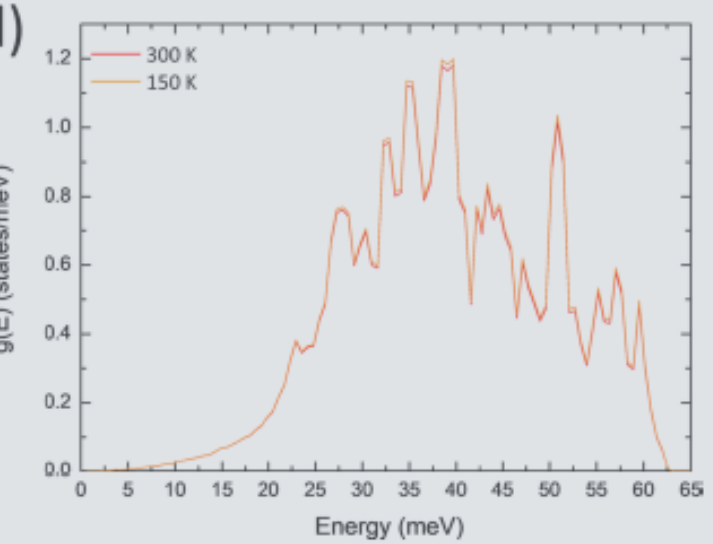

Figure 6. Experimental GVDOS of sample S1 (bulk) upon temperature variations measured with the TOF spectrometers (a) IN4 and (b) IN5. The theoretical neutron weighted GVDOS is also given for (c) IN4 and (d) IN5 experimental configurations at the same temperatures.

upshift of peaks in the high energy range $>35 \mathrm{meV}$ upon cooling. The small lowering of the intensity of the phonon DOS with increasing temperature to $300 \mathrm{~K}$ in the $\mathbb{I N} 4$ data should be due to the Debye-Waller contribution, as confirmed by our DFT calculations taking into account this effect as a function of temperature (Figure 6c). Our calculations also indicate that the effect of the Debye-Waller must be negligible in the IN5 data because of the much smaller Q space covering in the IN5 data compared to the IN4 data (Figure 6d).

The weak temperature effect on the phonon properties marks the S1 sample as a moderately quasi-harmonic crystal. This result is in line with the lattice thermal conductivity data.

3.3. Lattice Dynamics of Nanostructured $\mathrm{CrSi}_{2}$. The effect of the nanostructuring has been investigated by determining the cumulative thermal conductivity by DFT calculations. The results are shown in Figure 7 as a function of the phonon mean free path for direction perpendicular and colinear to the optical axis. One notices that phonons with a mean free path higher than $20 \mathrm{~nm}$ contribute to $50 \%$ of the lattice thermal conductivity. At enough high temperature such as $300 \mathrm{~K}$ and higher temperatures, the dominant scattering mechanism is the Umklapp scattering, which is considered in our DFT calculations, whereas other scattering mechanisms such as point defect scattering and electron-phonon scattering, not studied here, must be considered at lower temperatures. Thus, at high enough temperature, the nanostructuring down to $20 \mathrm{~nm}$ could decrease the lattice

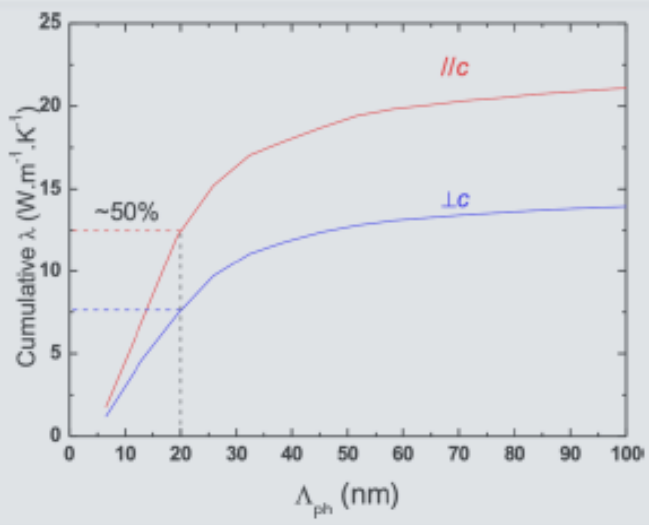

Figure 7. Cumulative thermal conductivity as a function of the phonon mean free path for directions perpendicular and collinear to the optical axis.

contribution to the thermal conductivity by about $50 \%$ if one does not consider the additional scattering mechanisms evoked above.

The experimental GVDOS measured at $300 \mathrm{~K}$ on bulk (S1) and nanocrystalline samples (S2 and S3) is given in Figure 8. A slight optical mode broadening and a downshift of $\sim 1 \mathrm{meV}$ are observed. In addition, an increase in the GVDOS is visible below $25 \mathrm{meV}$ for the S2 nanocrystalline samples (14 nm and 


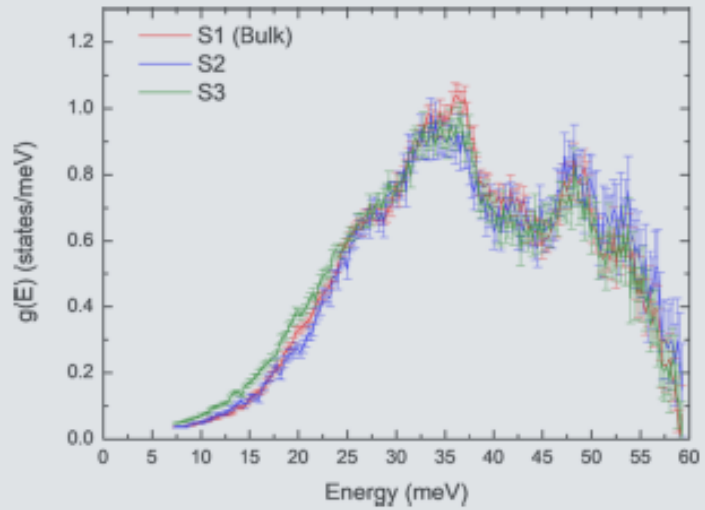

Figure 8. Experimental GVDOS for bulk (S1) and nanocrystalline samples (S2 and S3) at $300 \mathrm{~K}$ measured using the IN4 spectrometer.

$0.87 \%$ microstrain). This energy range corresponds to the acoustic branches of $\mathrm{CrSi}_{2}$ as described in Figure 4. These observations could be explained by a decrease in the Debye temperature and speed of sound upon nanostructuring $\mathrm{CrSi}_{2}$.

Within the incoherent scattering approximation, the Debye temperatures can be determined according to ${ }^{35}$

$$
\lim _{E \rightarrow 0} \frac{g(E)}{E^{2}}=\frac{9 N}{\theta_{\mathrm{D}}^{3} k_{B}^{3}}
$$

where $g(E)$ is the phonon GVDOS, $E$ is the phonon energy, $N$ is the number of atoms per unit cell $\left(N=9 \mathrm{in} \mathrm{CrSi}_{2}\right), k_{B}$ is the Boltzmann constant, and $\theta_{\mathrm{D}}$ is the Debye temperature. The sound velocity, $v_{s}$, can be also calculated by the following relation: ${ }^{36}$

$$
v_{\mathrm{s}}=\frac{\theta_{\mathrm{D}} k_{\mathrm{B}}}{\hbar}\left(\frac{6 \pi^{2} N}{V}\right)^{-1 / 3}
$$

where $V$ is the unit cell volume ( $V=108 \AA^{3}$ for this sample) and $\hbar$ is the reduced Planck constant. Nevertheless, as $\mathrm{CrSi}_{2}$ is made of coherent scatters, the GVDOS does not reflect the true velocity of sound. However, qualitative comparisons can be made between the samples. The calculated $\theta_{\mathrm{D}}$ values are given in Table 3 with their corresponding sound velocity.

Table 3. Apparent Debye Temperature and Apparent Sound Velocity of Bulk and Nanostructured Samples Determined from eqs 3 and 4) and Compared to the True Debye Temperature and True Sound Velocity of the Bulk Sample, both Experimental ${ }^{14}$ and Calculated ${ }^{5}$

\begin{tabular}{lcc}
\multicolumn{1}{c}{ sample } & $\theta_{\mathrm{D}}(\mathrm{K})$ & $v_{x}\left(\mathrm{~m} \cdot \mathrm{s}^{-1}\right)$ \\
bulk exp. ${ }^{14}$ & 793 & 6098 \\
bulk calcs & 766 & 5898 \\
S1 $($ bulk $)$ & 640 & 4920 \\
S2 $(64 \mathrm{~nm})$ & 622 & 4786 \\
S3 $(14 \mathrm{~nm})$ & 558 & 4296
\end{tabular}

The plot of the reduced density of states, $g(E) / E^{2}$, is shown in Figure 9. A strong increase in $g(E) / E^{2}$ at low energy is observed for the nanostructured sample S3 compared to S1 and S2. This further indicates that the apparent Debye temperature decreases from 640 to $558 \mathrm{~K}$ when the crystallite size in $\mathrm{CrSi}_{2}$ is decreased to $\sim 14 \mathrm{~nm}$ with microstrains as high

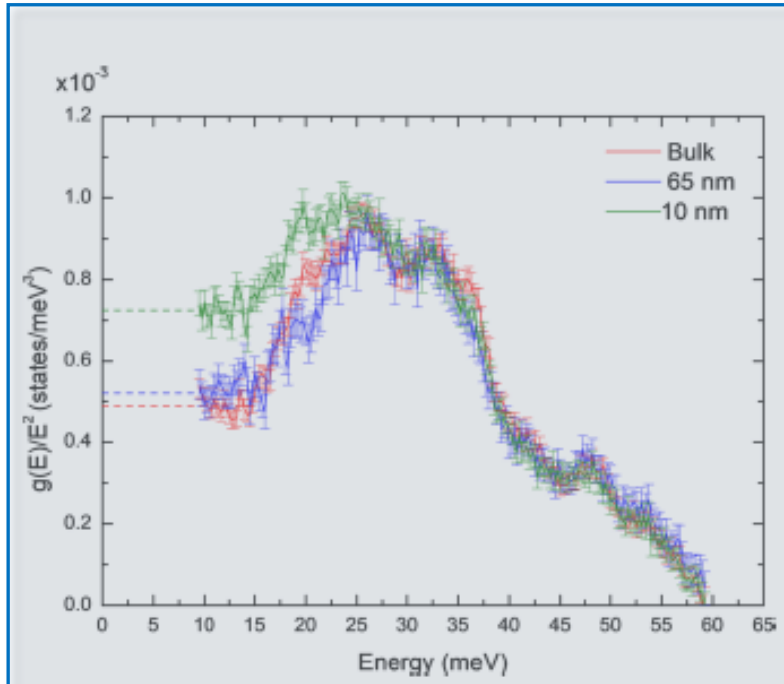

Figure 9. Reduced density of states of bulk (S1) and nanocrystalline $\mathrm{CrSi}_{2}$ (S2 and S3) at $300 \mathrm{~K}$ (from the IN4 beam line). The dotted lines indicate the limit value at low energy used for the Debye temperature calculation.

as $0.87 \%$ (sample S3). In contrast, nanostructuring at $64 \mathrm{~nm}$ with microstrains of $0.75 \%$ (sample S2) has a noticeable but limited impact $\left(\theta_{\mathrm{D}}=622 \mathrm{~K}\right)$.

As expected, the apparent sound velocity decreases with decreasing the crystallite size and increasing the intemal strain from $4920 \mathrm{~ms}^{-1}$ for bulk sample S1 to 4786 and $4296 \mathrm{~m} \cdot \mathrm{s}^{-1}$ for S2 and S3, respectively. A reduction of the thermal conductivity can therefore be expected below $64 \mathrm{~nm}$ as observed previously on dense pellets with a crystallite size of $\sim 50 \mathrm{~nm}$. ${ }^{\circ}$ This effect can be attributed to an increase in the grain boundaries together with an increase in the internal strains from the milling process.

3.4. Thermal Conductivity. The temperature dependence of the total thermal conductivity of the single crystal measured perpendicular to the $c$ axis (along $b^{*}$ ) below $300 \mathrm{~K}$ is shown in Figure 10. The values near $300 \mathrm{~K}$ are in good agreement with those reported in ref 37 . The phonon contribution to the total

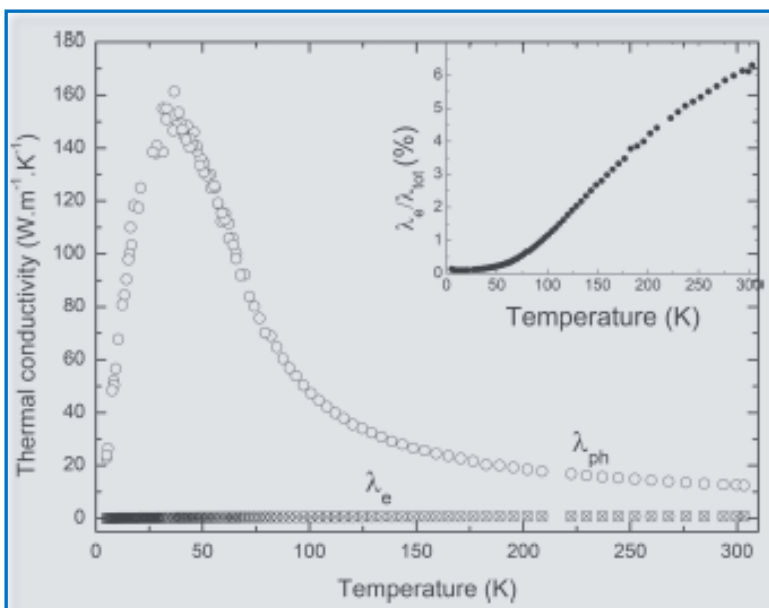

Figure 10. Temperature dependence of the total thermal conductivity of single-crystalline $\mathrm{CrSi}_{2}$ (S0) measured perpendicular to the $c$ axis. The ratio between $\lambda_{e}$ and $\lambda_{\text {bat }}$ is given in the inset assuming that $L=$ $L_{0}$. 
a)

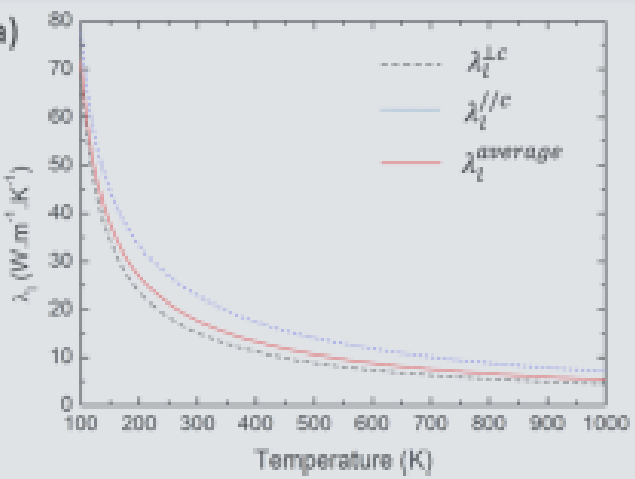

b)

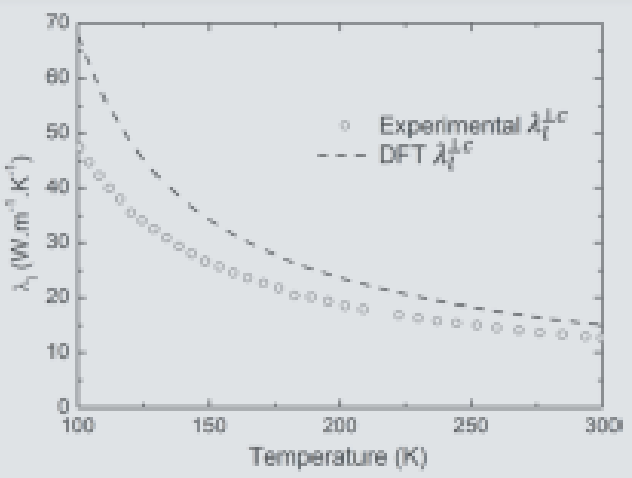

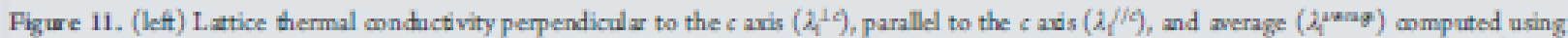
DFT calculations: (right) comnurison between the emerimental and thooretical lattice thermal conductivitv nemendicular to the $c$ aris $\lambda^{1}$.

thermal conductivity has been obtained by subtracting the electronic contribution calcubted using the WiedemannFranz law:

$$
\lambda_{e}=\frac{L T}{\rho}
$$

where $L$ is the Lorenz number and $\rho$ is the electrical resistivity. The nondegenerate nature of the hole gas, due to the semiconducting behavior of $\mathrm{CrSi}_{2}$, indicates significant deviations of $L$ from its degenerate limit $L_{0}=2.445 .10^{-8} \mathrm{~V}^{2}$ $\mathrm{K}^{-2}$. In nond egenerate semiconductors, $L$ is usually lower than $L_{0}$ when acoustic phonons or neutral impurity scattering dominates the scattering mechanisms. Nevertheless, assuming $L=L_{0}$, that is, assuming that $L$ reaches its upper limit, the battice contribution corresponds to more than $95 \%$ of the heat transport in $\mathrm{Cr}_{\mathrm{Si}}$ due to the negligible electronic contribution (Figure 10). Hence, in the present case, we can safely assume that $\lambda_{\text {oot }} \approx \lambda_{\text {. }}$. Some empirical studies have reported that the temperature of the maximum thermal conductivity is equal to $\sim 0.05 \theta_{\mathrm{D}}{ }^{35}$ In our case, the maximum thermal conductivity is found to be equal to $39.5 \mathrm{~K}$, which would give a Debye temperature of $790 \mathrm{~K}$, rather similar to that of $793 \mathrm{~K}$ obtained by Nalamura ${ }^{14}$ and by DFT calculation. ${ }^{5}$

The bittice thermal conductivity $\lambda_{4}$ has been computed using DFT calcubtions (see Figure 11). The bttice thermal conductivity in the plane perpendicular to $c\left(\lambda_{l}{ }^{1}\right)$ is dightly brger than our experimental values by about $20-30 \%$ in the $100-300 \mathrm{~K}$ range but is similar to the experimental results of Voronov et al at $300 \mathrm{~K}$. We note that recent DFT calculations ${ }^{13}$ could not reproduce the experimental values. The difference between our experimental and theoretical results with the literature dat $a^{3}$ can be explained by the significant contribution of the defects to the phonon scattering in $\mathrm{CrSi}_{2}$, which can strongly vary from one sample to another. Indeed, it is well known that $\mathrm{CrSi}_{2}$ can naturally contain high point defect concentration due to its homogeneity range extending between $\mathrm{CrSi}_{1.6}$ and $\mathrm{CrSi}_{203}{ }^{37,20}$ This inherent defect chemistry explains the high charge carrier concentrations $\left(0.5-1 \times 10^{21} \mathrm{~cm}^{3}\right)^{37,39,46}$ reported in prior studies. Such large amounts of defects should contribute to increase both point-defect and electron-phonon scattering.

\section{CONCLUSIONS}

The dispersion curves and the GVDOS of $\mathrm{CrSi}_{2}$ were determined by INS, showing a very good agreement with DFT calculations. Our findings reveal that the optical phonon contributes between 50 and $70 \%$ of the battice thermal conductivity, in contrast to what is usually admitted in most materials in which acoustic phonons are predominant. The so und velocity obtained from the phonon group velocity also shows a good agreement with both DFT calculations and ultrasonic experiments. ${ }^{14}$ The weak temperature dependence of the phonon properties of $\mathrm{CrSi}_{2}$ suggests that it is a moderately quas-harmonic crystal The GVDOS measured on nanostructured $\mathrm{CrSi} \mathrm{i}_{2}$ shows that the reduction of the crystallite size leads to a decrease in the Debye Temperature and in the sound velocity of $2.7 \%$ already at $64 \mathrm{~nm}(0.75 \%$ internal strain). This effect is even more pronounced upon decreasing the crystallite size to $14 \mathrm{~nm}(0.87 \%$ intemal strain) with a decrease in the sound velocity of $12.7 \%$. Consequently, the bttice contribution to the total thermal conductivity can be reduced, explaining what was observed previously for $50 \mathrm{~nm}$ dense $\mathrm{CrSi}_{2}$ pellets ${ }^{6}$ The analysis of the thermal conductivity of single-crystalline $\mathrm{CrSi}_{2}$ enables one to evaluate the Debye temperature of $\mathrm{CrSi}_{2}$, yielding a value of $790 \mathrm{~K}$, in good agreement with previous studies.

\section{ABBREVIATIONS}

GVDOS generalized vibrational density of state

HRTEM high-resolution transmission electron microscopy

SEM scanning electron microscopy

DFT density functional theory

INS inelastic neutron scattering

TOF time of flight

TAS triple axis spectrometer

EBSD electron back scattering diffraction 


\section{REFERENCES}

(1) Yang I.; Chen, Z. G-; Dargusch, M. S-; Zou, J- High Performanas Thermoelectric Materials: Progress and Thar Applia. tions. Adv. Energy Mater. 2018, 8, 1-28.

(2) Rowe, D. M. Thermolectric Handbook - Macro to Nano; CRC Press, Taylor and Francis, 2006.

(3) Burkov, A T. Silicide Thermoelectrias: Materials for Energy Harvesting. Phys Statue Solidi Appl. Mater. Sai. 2018, 215, 1-19.

(4) Kanuppaiah, S; Beaudhuin, M.; Vennoir, R. Investigation on the Thermoelectric Properties of Nanostructured Crl-XTisi2. J. Solid State Chem. 2013, 199, 90-95.

(5) Hermet, P.; Khalil, M.; Viennois, R; Beadhain, M.; Boungogne, D.; Ravot, D. Revisited Phonon Assignment and Electro-Mechanical Properties of Chromium Disilicide. RSC Adv 2015, 5, 19106-19116.

(6) Khalil, M-; Moll, A; Godfroy, M, Letrouit Lebranchu, A; Villeroy, B; Alleno, E.; Viennois, R.; Besudhuin, M. Thermoelectric Properties and Stability of Nanostructured Chromium Dasilicide CrSi2 J. Appl. Phys. 2019, 126, 135103.

(7) Godfroy, M; Khalil, M.; Niebel, C; Jarrosson, T-; Foir, D; Fland, V.; Serein Spiran, F-; Viennois, R.; Granier, M.; Beaudhuin, M. Transition Metal Silicide Surface Gratting by Multiple Functional Groups and Green Optimization by Mechanochemistry. Phys. Chem Chem. Phys. 2019, 21, 25720-25727.

(8) Godfroy, M.; Rusvel, A.; Mercier, F-; Grania, M.; Jarrosson, T.; Niebel. C. Serein Spirau. F-4 Viennois, R.; Beaudhuin M. Nano crystalline Chromium Dirilicide Synthesized by a Fast ChlorineTransfer-Reaction Mater. Lett. 2019, 247, 7-10.

(9) Chaix Pluchery, O.; Lacazeau, G. Vibrational Study of Transition Metal Disilidides, MSi2 (M-Nb, Ta, V, Cr). J. Raman Spectrose 1998, 29, 159-164.

(10) Lange, H.; Giehler, M-; Henrion, W-; Fenske, F-; Sieber, L; Oertel, G. Growth and Optical Characterization of $\mathrm{CrSi2}$ Thin Flms. Phys. status solidi 1992, 171, 63-76.

(11) Bellani, V; Guinzati, G; Marabdli, P; Piaggi, A; Borgheri, A.; Nav2, F.; Antonov, V. N.; Antonov, V. N.; Jepren, O.; et al. Theory and Experiment on the Optical Properties OfCrS12. Phys. Rev. B 1992, $46,9380-9389$.

(12) Borghesi, A.; Pisggi, A.; Franchini, A.; Guiznetti, G.; Nava, F-; Santoro, G. Far-Infrared Vihrational Spectrosopy in Crsi2. EPL. $1990,11,61-65$.

(13) Nakasawa, H.; Hayashi, K.; Talamatsı, T.; Mryazabi, Y. Lattice Dynamics and Lattice Thermal Conductivity of CrSi2 Calculated from First Principles and the Phonon Boltzmann Transport Equation. J. Appl Phys. 2019, 126, No 025105 .

(14) Nakamura, M. Elastic Constants of Some Transition Metal. Dirilidide Single Crystals. Metall. Mater. Trans A 1994, 25, 331-340.

(15) Kasa, M. M; Leithe Jasper, A-; Rosner, H; Schnelle, W-; Mutka, H_; Johnson, M. R-; Grin, Y. Vhrational Dynamics of the Filled Shutterudte Yb1-XFe 4Sb 12: Debye-Waller Factor, General. ired Density of States, and Flastic Structure Factor. Phys. Rev. B 2014, $89,1-8$

(16) Christensen, M; Abrahamsen, A. B; Christensen, N. B.; Jur anyi, $\mathrm{P}_{\text {; }}$ Andersen, N. H.; Lefmann, K.; Andreasson, J-; Bahl, C. R.; Iversen, B. B. Avoided Crossing of Rattler Modes in Thermoelectric Materials. Nat Mater. 2008, 7, 811-815.

(17) Kora, M. M.; Boehm, M.; Sischka, E; Schnelle, W.; Mutka, H.; Leithe Jasper, A. Low-Enengy Phonon Dispersion in LaFetSbl2 Phys Rev B 2015, 91, 1-8.
(18) Claudio, T.; Bessas, D; Birke, C. S.; Kieshich, G.; Panthöfer, M.; Sergueev, L; Tremel, W.; Hermann, R. P. Enhanced Debye Level in Nano $\mathrm{Zn} 1+\mathbf{S h}$, FeSb2, and NiSb: Nuclear Inelastic Spectroscopy On121 Sh. Phys. Status Sdidi Baric Res. 2014, 251, 919-923.

(19) Klobes, B; Bessas, D; Juranyi, P-; Görlitz, H.; Pacheso, V.; Hermann, R. P. Effect of Nanocrystallinity on Lattice Dynamics in Bi2Te3 Based Thermoelectrica. Phys Status Solidi - Rapid Res Lett 2015, 9, 57-61.

(20) Moll, A.; Laborde, S-; Barou, F-; Beaudhain, M. Centimetric CrSi2 Crystal Grown by the Vertical Gradient Freeze Method. J. Crost. Growth 2020, 534, No. 125505 .

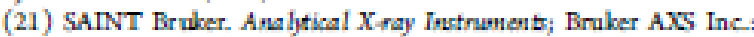
Madison, Wisconsin, USA, 2012

(22) SADARS Bruker. Analytical X-ray Instrumerts; Bruker AXS Inc, Madison, Wisconsin, USA, 2012

(23) SHEL.XTL. Bruker. Analytical Xray Instument; Bruker AXS Inc, Madison, Wisconsin, USA, 2012

(24) Rodriguez Carvajal, J. FUL.L.PROF: A Program for Rietveld Refinement and Pattern Matching Analysic. Satellite masting on poweder diffraction of the XV congress of the IUCr; Toulouse, France, 1990.

(25) L.AMP, the Lange Array Maripulation Program. Http://Www. IIl.Fr/Data treat/Lamp/ProntHtml.

(26) Kresse, G; Joubert, D. From Ultrasoft Pseudopotentials to the Projector Augmented Wave Method Phys. Rev. B 1999, 59, 17581775.

(27) Perdew, J. P.; Burke, K.; Ernzerhof, M. Generalized Gradient Approumation Made Simple. Phys. Rev. Lett 1996, 77, 3865-3868.

(28) Hermet, P-; Jund, P. Iattice Thermal Conductivity of NTiSn Half Heusler Thermoeleatric Materials from Finst Principles Calcu lations. J. Alloys Compd 2016, 688, 248-252

(29) Claudio, T.; Schierning G.; Theismann, R; Wiggas, H.; Schober, H.; Kozz, M. M.; Hermann, R. P. Effocts of Impurities on the Lattice Dynamics of Nanocrystalline Silicon for Thermoelectric Application. J. Mater. Sci 2013, 48, 2836-2S45.

(30) Slack, G. A. The Thermal Conductivity of Nonmetallic Crvstak Solid Stat. Phvs 1979. 34. 1-71

(31) Shigz, T-; Aketo, D.; Feng, I.; Shiomi, J. Harmonic Phonon Theory for Calculating Thermal Conductivity Spectrum from FirstPrinciples Dispersion Relations. AppL Phys. Latt. 2016, 108, No. 4950851 .

(32) Callaway, J. Model for Lattice Thermal Conductivity at Low Temperatures. Phys. Rev. 1959, 113, 1046.

(33) Blundell, S. J-; Blundell, K. M. Concepts in Thermal Physics Orford Uni; 2006

(34) Kittel, C. Phyrigue de Fetat Solide; Dunod, 1998.

(35) Sladx, G. A; Galginaitis, S. Thermal Conductivity and Phonon Scattering by Magnetic Impurities in CdTe. Phys Rev. 1964, 133, No. $A 253$

(36) Holland, M. G. Analysis of Lattice Thermal Conductivity. Phys. Rev. 1963, 132, 2461-2471.

(37) Voronov, B. K.; Dudlin, L. D.; Trusova, N. N. The Features of Physical Chemical Structure of Chromiun Disilicide (in Russian); Khimicherkaya Svjaz v Poluprovodn Nauk i Tellinilea, Minsk, 1969 291

(38) Matthiessen, A; Von Bose, M. On the Influence of Temperature on the Electric Conducting Power of Metals. Philos. Trane. 1862, 152, 1-27.

(39) Asen Palmer, M-; Barthowshi, K.; Gmelin, E-; Cardona, M.; Zhernov, A.; Inyushkin, A.; Taldenkov, A.; Ozhogin, V. Thermal Conductivity of Germanium Crystals with Different Isotopic Compositions. Phys. Rev. B 1997, 56, 9431-9447.

(40) Peierls, V. R. Zur Kinetischen Theorie Der Warmeleitung in Kristallen. Ann Phys. 1929, 395, 1055-1 101. 